\title{
A temporal QoS based CPU scheduling model for multimedia applications in general purpose operating systems
}

\author{
D. Bourges Waldegg \\ ENST Bretagne, Networks and Multimedia Department \\ BP 78, 35512 Cesson-Sévigné Cedex, France \\ Tel. (33) 2991270 23, Fax (33) 299127030 \\ email: Daniela.Bourges@enst-bretagne.fr
}

\begin{abstract}
We present a CPU scheduling model aimed at satisfying multimedia presentation temporal QoS requirements. The model is based on a playout specification and a runtime application structure that allows workahead processing, and quality degradation for delay or overload situations.
\end{abstract}

Keywords

Operating system QoS support for multimedia, synchronization, CPU scheduling.

\section{INTRODUCTION}

Multimedia temporal QoS requirements include both individual stream's continuity and inter-stream synchronization. In order for an operating system to manage these QoS requirements, its CPU scheduler needs an explicit knowledge of data temporal characteristics to perform a scheduling aimed at respecting them. This is not the case in existing general purpose OSs (Nieh et al. 1993). Currently, application developers have to define application-specific temporal QoS enforcement methods which can result in unacceptable presentation quality, since CPU scheduling is decoupled from decisions taken within these methods (Bourges Waldegg et al. 1996).

In a general purpose OS, users may accept a degraded quality presentation under varying load conditions. Integrating synchronization and quality adaptation into the system scheduler can help to take more accurate decisions and to make a better use of the CPU resource. This integration also provides programmers with a generic support which they can use to build multimedia applications.

So we have developed a CPU scheduling model for adaptive multimedia presentation applications, that integrates synchronization and adaptation support. The model is composed of an application structure aimed at minimizing degradation for streams with intensive-CPU needs; a QoS and playout scenario specification to transmit QoS requirements to the kernel; and an on-line scheduling algorithm.

Building QoS into Distributed Systems A. Campbell \& K. Nahrstedt (Eds.)

(C) 1997 IFIP. Published by Chapman \& Hall 


\section{QOS BASED SCHEDULING MODEL}

We will briefly describe the components of our scheduling model. For a more detailed description please refer to (Bourges Waldegg 1997).

\section{Presentation runtime structure}

During a multimedia presentation, data units (DUs) composing continuous media streams are obtained from network or disk. Before they can be presented, DUs may need a more or less important amount of processing. Continuity of a stream is ensured by the sequential presentation of DUs at periodic instants.

Note that only the DU's presentation needs strictly periodic attention. Reading and processing of a DU can be anticipated and further performed in a workahead basis if CPU time is available, absorbing network delay and processing time variations, minimizing thus presentation deadline violations.

Data must be manipulated by adapted execution structures (the scheduling units in the kernel, which we call threads). We propose an execution model that performs each activity (reading, processing and presentation or degradation) in an individual thread, an application consists then of a set of these threads for each stream.

\section{QoS parameters and playout scenario description}

The temporal QoS specification of a multimedia presentation application includes parameters such as individual streams' rates, tolerable jitter for DU presentation, and tolerable skew between streams.

For describing the playout scenario, we use the Timed Stream Petri Net (TSPN) model (Sénac et al. 1994). This model is a timed extension of Petri Nets, which adds firing intervals to arcs outgoing from places. A continuous media stream is then modelled by a sequential composition of places and transitions, firing intervals allow for the expression of jitter and variable processing times. Typed inter-stream synchronization points are modelled by transitions with several input arcs. Figure 1 shows an example of a TSPN modelling an audio/video presentation.

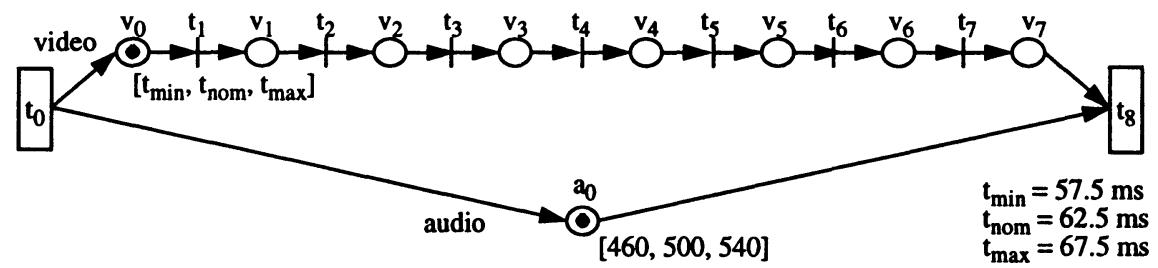

Figure 1 Example of a TSPN.

\section{On-line CPU Scheduling Algorithm}

At initialization, the application transmits its QoS parameters and playout scenario to the kernel. The scheduler then executes the TSPN representing the application.

If a transition is firable at scheduling instant $\theta$, presentation threads corresponding to all streams involved in the transition are scheduled in internal priority order (which reflects the importance of the stream for inter-stream synchronization). If a presentation thread is not ready, a degradation thread is scheduled.

If there's no firable transition at instant $\theta$, reading/processing threads in non- 
workahead state are scheduled in deadline order, the deadline being the lower bound of the dynamic temporal validity interval corresponding to the associated arc (whose values depend on the static interval and execution state). Reading/processing threads are scheduled for a duration of one time quantum, a value which reflects processing needs for each stream.

If at instant $\theta$ there are no reading/processing threads in nonworkahead state, workahead threads can start to be scheduled in a round robin basis.

In summary, the decision algorithm will schedule in priority:

- presentation threads when scheduling instant belongs to firing interval, in internal priority order;

- nonworkahead reading/processing threads in deadline order;

- workahead processing/reading threads in round-robin.

\section{EXAMPLE}

We illustrate our scheduling model with an audio/video software decoding/presentation application. Let us consider QoS demands as follows: 16 images/s, maximum skew between audio and video $=80 \mathrm{~ms}$. The TSPN depicted in Figure 1 corresponds to this QoS specification, with a synchronization interval of half a second. The audio stream, which is more sensible to degradation than the video flow, is set as the master stream for inter-stream transitions. As video processing is more CPU demanding than audio processing, we fix the time quantum for video to be $30 \mathrm{~ms}$, and the time quantum for audio to be $5 \mathrm{~ms}$.

We consider that video images need the following reading/processing times: $\mathrm{v}_{1}=120 \mathrm{~ms}, \mathrm{v}_{2}=45 \mathrm{~ms}, \mathrm{v}_{3}=45 \mathrm{~ms}, \mathrm{v}_{4}=80 \mathrm{~ms}, \mathrm{v}_{5}=45 \mathrm{~ms}, \mathrm{v}_{6}=45 \mathrm{~ms}$ and $\mathrm{v}_{7}=120$ ms. Presentation threads need $10 \mathrm{~ms}$ each time an image is displayed or an audio block is sent to device. The audio block needs $40 \mathrm{~ms}$ to be processed. Note that these values are not known a-priori by the scheduler. We consider also that processing of image $v_{1}$ had been scheduled in a workahead basis, and at the time the synchronization interval starts, it has progressed $110 \mathrm{~ms}$.

The schedule obtained with our algorithm is shown in Figure 2. Note that all video images excepting image $v_{7}$ are presented within their validity intervals even though some of their processing times exceed the stream's period. Image $v_{7}$ was not ready at time transition $t_{7}$ needed to be fired $(\theta=427.5 \mathrm{~ms})$. A video degradation thread was thus scheduled, the degradation policy consists of just rejecting the image, and advancing to the reading/processing of the next image. At the end of the synchronization interval, audio block $a_{1}$ is ready for presentation, and inter-stream transition $\mathrm{t}_{8}$ is fired at time $\theta=472.5$, which is a temporally correct value.

\section{CONCLUSION AND PERSPECTIVES}

We have presented a QoS based scheduling model for adaptive multimedia presentation applications, which offers a support for synchronization and adaptation at a lower level, saving thus decision layers that can cause inaccuracy. Degradation decided by the scheduler is useful for a better resource utilization. Finally, with our runtime structure we allow for anticipation and workahead processing that can be useful 


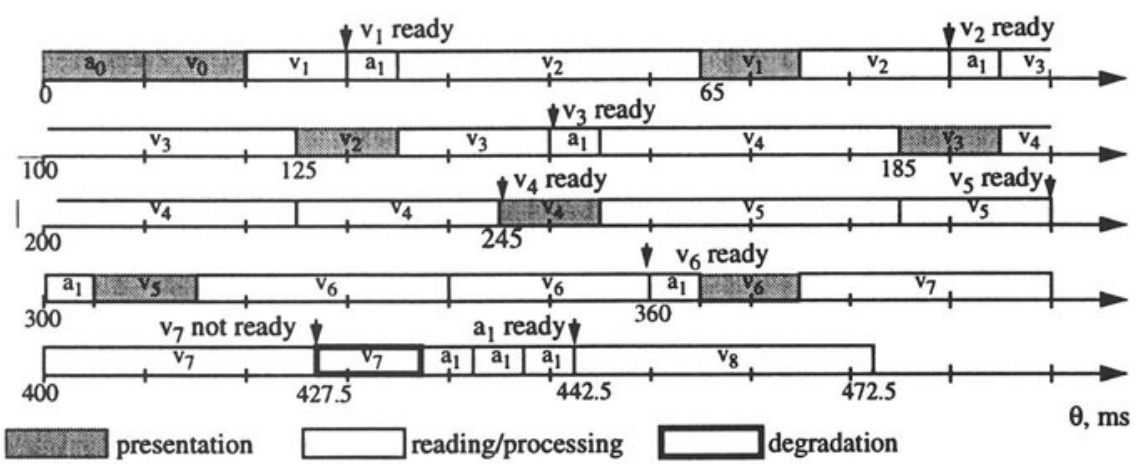

Figure 2 MPEG audio/video schedule.

in overload situations.

We are currently working on the implementation of our model in the Solaris operating system. Problems related to priority calculation costs are being analyzed.

\section{REFERENCES}

Bourges Waldegg, D., Lagha, N. and Le Narzul, J.P. Multimedia Applications on a UNIX SVR4 Kernel: Performance Study. In Proceedings of the 3rd International COST 237 Workshop on Multimedia Telecommunications and Applications, Barcelona, Spain, November 1996.

Bourges Waldegg, D. A CPU Scheduling Model for Respecting Temporal QoS in General Purpose Operating Systems. ENST Bretagne Technical Report, 1997.

Nieh, J., Hanko, J.C., Northcutt, J.D. and Wall, G.A. - SVR4 UNIX Scheduler Unacceptable for Multimedia Applications. In Proceedings of the 4th International Workshop on Network and Operating System Support for Digital Audio and Video, Lancaster, UK, November 1993.

Sénac, P., Diaz, M. and de Saqui-Sannes, Pierre. Towards a Formal Specification of Multimedia Synchronization Scenarios. Annals of Telecommunications, Vol. $49, N^{\circ} 5-6,1994$.

\section{BIOGRAPHY}

Daniela Bourges Waldegg was born in Mexico City, in 1970. She obtained an Electrical Engineering degree in 1993 from the "Universidad Autónoma Metropolitana", in Mexico City and a Computer Science "Diplôme d'Etudes Approfondies" from "Université de Rennes 1" in 1994. She is currently a PhD student at the Networks and Multimedia Department of the "Ecole Nationale Supérieure des Télécommunications de Bretagne" in Rennes, France, supported by a grant from the Mexican National Council for Science and Technology (CONACyT). 